\title{
Decreased neutrophil apoptosis in tracheal fluids of preterm infants at risk of chronic lung disease
}

\author{
J Oei, K Lui, H Wang, R Henry
}

Arch Dis Child Fetal Neonatal Ed 2003;88:F245-F249

See end of article for authors' affiliations

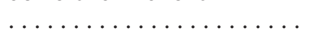

Correspondence to: Dr Lui, Department of Newborn Care, Royal Hospital for Women, Barker Street, Randwick, NSW, Australia 2031; k.lui@unsw.edu.au

Accepted 20 August 2002

\begin{abstract}
Objective: To investigate the hypothesis that preterm infants who are more susceptible to lung damage have decreased neutrophil apoptosis, and to explore its relation to interleukin 10 (IL 10) concentration. Design: Prospective cohort design.

Patients: One hundred tracheal fluid specimens from 50 week-1 ventilated infants were examined for IL 10 (by enzyme linked immunosorbent assay) and neutrophil apoptosis (by light microscopy).

Results: Neutrophil apoptosis was absent or less than $0.22 \%$ (median $0 \%$ ) in the 11 infants with chronic lung disease (CLD) (24-31 weeks gestation) during the first 4 days of life. This was significantly lower than that of the 20 preterm infants without CLD (27-31 weeks gestation; median $0.47 \%$, range $0-1.25 \%$ ) and 19 term infants (median $0.5 \%$, range $0-2.25 \%$ ). There was an increase in apoptosis in infants with CLD (median 0.44\%, $p=0.046$ ) during days 5-7. Few infants without CLD were intubated beyond 4 days. Median apoptosis on days $5-7$ was $0.26 \%$ and $2.78 \%$ for non-CLD preterm and term infants, but differences were not significant. IL 10 concentration in tracheal fluid of infants with CLD was less than $5 \mathrm{pg} / \mathrm{ml}$. None of the infants with IL 10 greater than $5 \mathrm{pg} / \mathrm{ml}$ developed CLD. The range of IL 10 concentrations in tracheal fluid from infants without CLD was wide (0-938 pg/ml). There was no apparent correlation between IL10 levels and percentage neutrophil apoptosis in infants without CLD. Conclusion: Preterm infants with low levels of IL 10 and neutrophil apoptosis may be predisposed to disordered lung repair. Further studies into the method of disposal of senescent neutrophils within preterm lungs are required.
\end{abstract}

$\mathrm{P}$ ositive pressure ventilation and oxygen disrupts lung epithelium. An inflammatory response characterised by a neutrophilic influx rapidly occurs. ${ }^{12}$ There is no evidence that these neutrophils are able to leave the lung and re-enter the microcirculation after the inflammatory stimulus is resolved. ${ }^{3}$ Their activity must thus be curtailed, and senescent neutrophils must be disposed of before healing can occur. Infants who develop chronic lung disease (CLD) have more alveolar neutrophils and cytotoxic byproducts such as elastase and leukotriene $\mathrm{B}_{4}$ than infants whose acute lung disease resolves. ${ }^{4}$

How neutrophils are cleared from the lungs of premature infants is unknown. Senescent neutrophils that die by necrosis release toxic intracellular substances that may perpetuate lung damage. ${ }^{5}$ Apoptosis or programmed cell death, on the other hand, reduces the inflammatory activity of both neutrophils and macrophages ${ }^{6}$ and has been shown to hasten the resolution of experimental lung injury. ${ }^{38}$ The only endogenous factor known to promote neutrophil apoptosis is interleukin (IL) 10, an anti-inflammatory cytokine present in low quantities in the lungs of patients with severe respiratory disease. ${ }^{9}$ We hypothesised that premature infants are more at risk of lung injury because of reduced neutrophil apoptosis and ILl0.

\section{METHODS}

Mechanically ventilated infants admitted to the neonatal intensive care unit of the Royal Hospital for Women were recruited into our study. We defined CLD in premature infants as a need for support with supplemental oxygen and/or mechanical ventilation beyond the postconceptional age of 36 weeks. ${ }^{10}$ Ventilatory strategies as well as decisions on treatment with exogenous surfactant and corticosteroids were determined by individual neonatologists. Our nursery administers exogenous surfactant (bovine; Survanta; Abbott Pharmaceuticals) as a rescue treatment in radiologically proven hyaline membrane disease (HMD) or meconium aspiration syndrome (MAS). Dexamethasone is prescribed in a tapering regimen of $4.5 \mathrm{mg} / \mathrm{kg}$ over two weeks for acute respiratory deterioration secondary to evolving CLD. Theophylline is prescribed to infants less than 30 weeks gestation for 12 hours before extubation to augment the success rate of extubation. ${ }^{11}$ This study was approved by the ethics committee of the South Eastern Sydney Area Health Service.

\section{Tracheal fluid collection}

Tracheal fluid was collected at least twice weekly during the first week of each infant's life. Collection was timed to coincide with laboratory availability (Mondays, Wednesdays, and Fridays) and with routine endotracheal suctioning of each intubated infant every six to eight hours. Two or more specimens were thus obtained from each infant depending on the day of its birth. Tracheal fluid was obtained in a similar fashion to that of McColm et $\mathrm{al}^{12}$ : we instilled $0.5 \mathrm{ml}$ normal saline into each infant's endotracheal tube and suctioned the residue with a 5F suction catheter after two or three ventilator breaths. Suction catheters were passed to a standardised length of $0.5-1 \mathrm{~cm}$ beyond the tip of each endotracheal tube. This method of suctioning was well tolerated by even the most critically unwell infants. Each collection was transported immediately to our on site laboratory for processing. Specimens that yielded less than $50 \mu \mathrm{L}$ lavage fluid were discarded because of a possibility that adjustment of low volume specimens would result in large assay errors.

Sample processing and analysis of apoptosis Cells were isolated by centrifugation and resuspension of tracheal fluid at $200 \mathrm{~g}$ for 10 minutes and then counted with

Abbreviations: CLD, chronic lung disease; IL, interleukin; HMD, hyaline membrane disease; MAS, meconium aspiration syndrome; TNF, tumour necrosis factor 


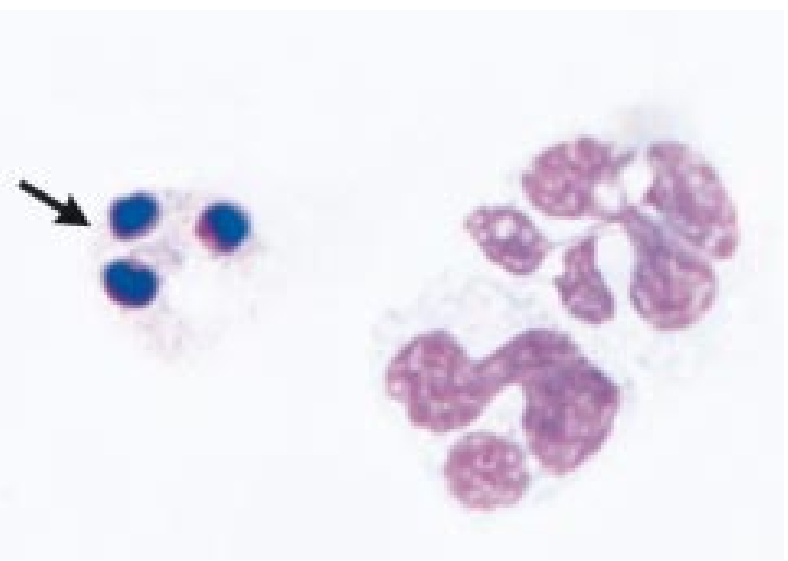

Figure 1 Neutrophil showing the characteristic changes of apoptosis (arrow) with densely condensed and rounded nuclear material.

a haemocytometer. Supernatant was stored at $-80^{\circ} \mathrm{C}$ and examined in batches for IL8 and IL10. Two or more slides were made by cytospin preparation of each sample, and the slides were stained with Diff-Quik (Lab Aids Pty Ltd, Narrabeen, Australia) and confirmed by agarose gel electrophoresis. This procedure involves the fixation of the cell pallet obtained immediately after centrifugation of the lavage material by $70 \%$ alcohol. To obtain DNA, the fixed cells were then treated by lysis and nuclear buffers. Ethidium bromide staining was used to ascertain if ladder development had occurred during agarose gel electrophoresis of the extracted DNA (concentration of agarose gel was $2 \%$; electrophoresis was performed for 30 minutes using a Gene Power Supply (Pharmacia GPS 200/400) at $100 \mathrm{~V}$ ).

Differential cell counts and characteristic changes in neutrophil apoptosis (chromatin condensation and simplification of nuclear structure $)^{6}$ were assessed by phase contrast microscopy of 400 cells under oil immersion (fig 1). Even though the TUNEL (terminal deoxynucleotidyl transferase mediated dUTP nick end labeling) method is widely used to identify apoptotic cell fractions in cultured cell preparations, we used Diff-Quik staining to avoid some of the potential artifacts associated with the TUNEL assay-for example, dependence on staining kinetics of the reagent, or on tissue fixation, or on the extent of proteolysis of the cells. ${ }^{13}$

\section{Cytokine analysis}

IL10 was analysed in batches by enzyme linked imuunosorbent assay (ELISA; R \& D, Minneapolis, Minnesota, USA). The minimum detectable level of this assay was $0.5 \mathrm{pg} / \mathrm{ml}$. The reproducibility of IL10 assays was confirmed by interlaboratory examination of seven selected tracheal fluid specimens (Airways Research Centre, John Hunter Hospital, Newcastle, Australia), as well as by the addition of anti-ILl0 immunoglobulin and exogenous human IL10 to several selected specimens. Cytokine values were expressed as weight/volume of lavage because no other satisfactory correction factor for tracheal fluid cytokine measurement is currently available. ${ }^{14}$

\section{Statistical analysis}

This was performed with the Mann-Whitney U, KruskalWallis, Student's $t$, and Fisher exact tests. $p<0.05$ was considered significant.

\section{RESULTS}

Of the 52 ventilated infants enrolled into our study, two died from necrotising enterocolitis before 36 weeks corrected age, and their results were excluded from our final analysis. Thirty one of the remaining 50 infants were preterm-that is, $<37$ weeks gestation. Most (23/31, 74\%) were ventilated for HMD. Table 1 summarises the clinical characteristics of all 50 infants. All HMD infants received at least one dose of exogenous bovine surfactant. None of the infants received corticosteroids during the first week even though most infants with CLD (8/11,73\%) and a term girl with MAS were treated with dexamethasone during or after the second week of life.

Two mothers had chorioamnionitis and both their infants developed CLD. Placental bacterial cultures were sterile in one and positive for group B streptococcus in the other. In our institution, low vaginal swabs are routinely performed on gravid women either at 28 weeks gestation or at delivery (if birth occurred before 28 weeks). Group B streptococcus was grown in the low vaginal swabs of four women (three with infants with CLD, and one with a term infant), and these women were treated with intrapartum penicillin. None of their infants developed positive blood cultures. A non-CLD preterm infant grew Enterococcus coli on blood collected immediately after birth. Her mother's low vaginal swab was negative.

The $11(36 \%)$ preterm infants who developed CLD were significantly more premature than the 20 preterm infants whose acute lung disease resolved, and the duration of ventilation was significantly longer (table 1). Two 28 week preterm infants without CLD were re-intubated at 52 and 21 days of age because of bronchiolitis and coagulase negative staphylococcus sepsis respectively. These infants were not oxygen dependent and both were easily extubated (into room air) after resolution of the acute illnesses.

None of the infants in our study were treated with dexamethasone during the time of specimen collection. Six infants (three with CLD and three without) were given theophylline before extubation, but no specimens were obtained from infants on concurrent theophylline treatment.

Of the 112 tracheal fluid specimens obtained, 12 (four from each group) were discarded because of low aspirate volume (less than $50 \mu \mathrm{L}$ ). Median tracheal fluid volume was $100 \mu \mathrm{L}$ (range 50-100) for CLD specimens, $110 \mu \mathrm{L}$ (range 50-450) for non-CLD preterm specimens, and $150 \mu \mathrm{L}$ (range 50-400) for term specimens. Cytokine and cell analysis were thus performed on 100 tracheal fluid samples (20 CLD, 44 non-CLD preterm, 36 term).

\section{Tracheal fluid leucocytes in relation to CLD}

There was a wide range of white cells in the tracheal fluid from all three groups. Median cell count was lowest in non-CLD preterm infants and highest in term infants, but overall values were not significantly different (median (range) white cell count: CLD, $4.3 \times 10^{6}$ cells/ml (0.15-15.8); non-CLD preterm, $2.9 \times 10^{6}$ cells $/ \mathrm{ml}(0-107)$; term, $7.6 \times 10^{6}$ cells $/ \mathrm{ml}(0-116.2)$, $\mathrm{p}=0.771)$. A term female infant with MAS on day $2(116.3 \times$ $10^{6}$ cells $\left./ \mathrm{ml}\right)$, a 28 week female infant with transient tachypnoea of the newborn on day $1\left(85.3 \times 10^{6} \mathrm{cells} / \mathrm{ml}\right)$, and a 32 week male infant with HMD on day $7\left(107 \times 10^{6}\right.$ cells $\left./ \mathrm{ml}\right)$ had the highest tracheal fluid white cell counts. The 32 week boy was extubated on day 8 , the 28 week girl on day 2 , and the term infant on day 11 . None of them were treated with steroids and none developed CLD.

Neutrophils were the predominant leucocytes in tracheal fluid. Non-CLD preterm infants had the lowest proportion of neutrophils (mean (SD) $66.4(41.8) \%$ ). Term infants had the highest $(85.8(24.6) \%, p=0.018)$. Infants with CLD had intermediate numbers of neutrophils $(70.9(31.3) \%)$, but this was not significantly different from the other two groups $(\mathrm{p}=0.712 v$ non-CLD preterm; $\mathrm{p}=0.11 v$ term $)$.

\section{Neutrophil apoptosis in relation to CLD and postnatal age}

Overall, term infants had a higher percentage of apoptotic neutrophils in week 1 (median $0.5 \%$, range $0-10.5 \%$ ) compared with either CLD (median $0.22 \%$, range $0-1.3 \%$; 
Table 1 Basic and clinical data of study infants

\begin{tabular}{|c|c|c|c|}
\hline & $\operatorname{CLD}(n=11)$ & $\begin{array}{l}\text { Preterm non-CLD } \\
(n=20)\end{array}$ & Term $(n=19)$ \\
\hline Gestational age (weeks)* & $27(24-31)$ & 31 (27-33) & $40(38-42)$ \\
\hline Birth weight $(\mathrm{g})^{*}$ & 813 (520-1500) & 1600 (830-2190) & 3640 (2220-4440) \\
\hline Male & $9(82 \%)$ & $14 / 20(70 \%)$ & $11 / 19(58 \%)$ \\
\hline Days ventilated* & \multicolumn{2}{|c|}{ Indications for ventilation } & $5(2-11)$ \\
\hline HMD & 9 & 14 & 5 \\
\hline TTN & 1 & 4 & 2 \\
\hline Apnoea & 1 & 2 & \\
\hline Meconium aspiration & 0 & 0 & 3 \\
\hline PPHN & 0 & 0 & 3 \\
\hline Myocarditis & 0 & 0 & 1 \\
\hline $\mathrm{CHD}$ & 0 & 0 & 1 \\
\hline HIE & 0 & 0 & 3 \\
\hline Sepsis & 0 & 0 & 1 \\
\hline \multicolumn{4}{|c|}{$\begin{array}{l}\text { Values are median (range) or number (\%). } \\
{ }^{*} \mathrm{p}<0.001 \text {, Kruskal-Wallis. } \\
\mathrm{CLD} \text {, Chronic lung disease; HMD, hyaline membrane disease; TTN, transient tachypnoea of the newborn; } \\
\text { PPHN, persistent pulmonary hypertension; CHD, congenital heart disease; HIE, hypoxic ischaemic } \\
\text { encephalopathy. }\end{array}$} \\
\hline
\end{tabular}

$\mathrm{p}=0.046$ ) or the combination of all preterm infants (median $0.26 \%$, range $0-1.5 \%$; $p=0.019$ ). The highest $(10.5 \%)$ was from a female infant with MAS from tracheal fluid specimens obtained on day 5 . Figure 2 summarises the results.

As most of the infants without CLD were extubated after the first 4 days of life, only a few tracheal fluid specimens were obtained from these infants during this period. Tracheal fluid results were thus divided into days $1-4$ and 5-7 for comparison. Some infants had two tracheal fluid specimens in either of the time epochs. To achieve equal weight for each infant in this analysis, the mean value of these was taken as the single individual value within the time epoch. Results remained consistent with the trend illustrated in fig 2. Neutrophil apoptosis was either absent or less than $0.22 \%$ in the 11 infants with CLD during the first 4 days of life (median 0\%).

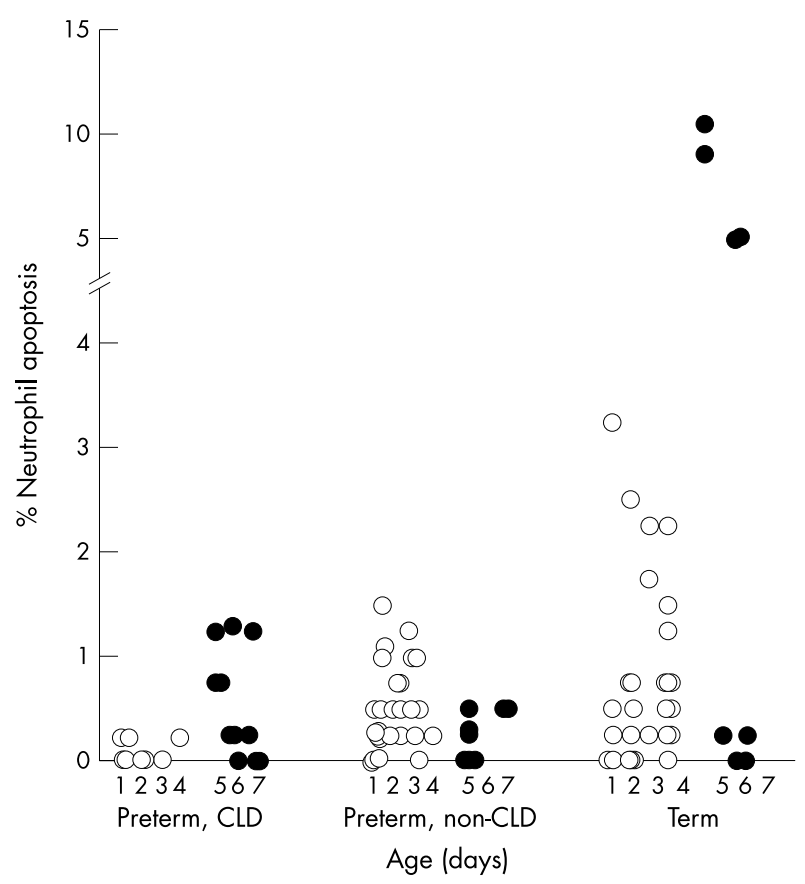

Figure 2 Percentage of neutrophils that are apoptotic in tracheal fluids of ventilated infants during the first week of life. Three groups of infants are shown: preterm infants with chronic lung disease (CLD), preterm infants without CLD, and term infants. Fewer tracheal fluid specimens were obtained beyond the first 4 days of life in non-CLD preterm and term infants (closed circles).
Compared with infants without CLD during the first 4 days, those with CLD had a lower percentage of apoptotic neutrophils (non-CLD preterm: median $0.47 \%$, range $0-1.25 \%$, $\mathrm{p}=0.001$, Mann-Whitney; term infants: median $0.5 \%$, range $0-3.25 \%, p=0.018)$. There was a trend towards an increase in the percentage of neutrophil apoptosis in infants with CLD during days $5-7$ (median $0.44 \%$, range $0-1.3 \%, \mathrm{p}=0.046$ ). Median apoptosis on days 5-7 for non-CLD preterm and term infants was $0.26 \%$ and $2.78 \%$ respectively, but differences did not reach significance.

\section{Neutrophil apoptosis in relation to IL10}

ILl0 concentration was less than $5 \mathrm{pg} / \mathrm{ml}$ in all CLD tracheal fluid specimens (fig 3). The range of IL10 from the non-CLD preterm specimens was much wider $(0-938 \mathrm{pg} / \mathrm{ml})$ and fluctuated throughout the first week of life. IL10 levels were greater than $5 \mathrm{pg} / \mathrm{ml}$ in 11 non-CLD preterm infants and in six term infants. Respiratory conditions in this group of infants were varied and ranged from those necessitating profound ventilatory support (such as term infants with MAS) to those with only mild disease (such as transient tachypnoea of the newborn) or no lung disease (such as infants intubated electively for seizure control).

The relation between IL10 and apoptosis was examined in non-CLD preterm and term infants because IL10 and neutrophil apoptosis levels were both low in infants with CLD. There was no apparent correlation between IL10 and apoptosis in either preterm $(r=0.128, \mathrm{p}=0.357, \mathrm{NS})$ or term $(r=0.212$, $\mathrm{p}=0.331, \mathrm{~ns})$ infants.

\section{DISCUSSION}

To our knowledge, our study is the first to examine both reduced neutrophil apoptosis and IL10 in the tracheal fluid of premature infants who subsequently develop CLD. IL10 inhibits the production of proinflammatory cytokines such as IL8, tumour necrosis factor (TNF) $\alpha$, and IL6 from neutrophils and macrophages. ${ }^{15} 16$ These cytokines are essential for maintaining inflammation. IL8, for example, is a powerful pulmonary neutrophil chemotaxin that acts in concert with TNF $\alpha$ to prolong neutrophil longevity by inhibiting apoptosis. ${ }^{17}{ }^{18}$ In a recent pilot study, we found no difference in tracheal fluid IL8 between infants with and without CLD, although IL8 tended to decrease towards the end of the first week in infants without CLD. ${ }^{19}$ There is, as yet, little evidence that neutrophils entering the lung as a result of injury are able to return to the microcirculation after dissipation of the injurious process. ${ }^{3}$ Therefore, for healing to occur, redundant neutrophils need to be inactivated and cleared from the lung. 


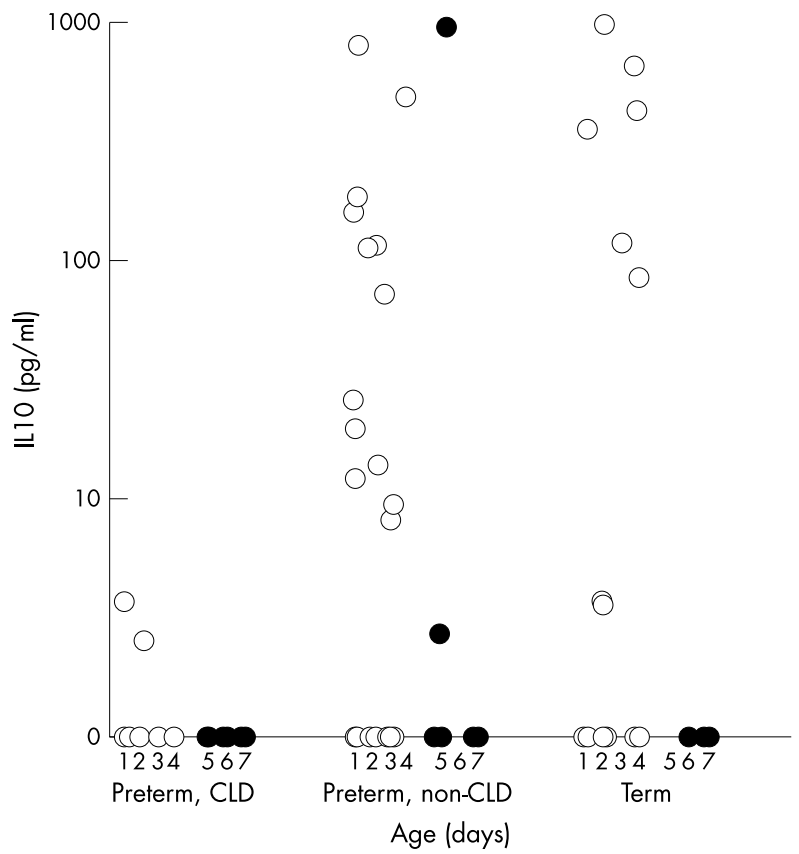

Figure 3 Interleukin 10 (IL1O) in tracheal fluids of ventilated infants during the first week of life. IL 10 concentrations are shown in $\log _{10}$ scale. Three groups of infants are shown: preterm infants with CLD, preterm without CLD, and term infants. Fewer tracheal fluid specimens were obtained beyond the first 4 days of life in non-CLD preterm and term infants (closed circles).

Apoptotic neutrophils lose the ability to degranulate potentially injurious intracellular contents. ${ }^{20}$ Macrophages that phagocytose apoptotic neutrophils do not release proinflammatory agents such as IL8, IL1 $\beta$, and TNF $\alpha .^{21}{ }^{22}$ Neutrophil apoptosis is thus an important mechanism by which various forms of acute lung injury are resolved..$^{93}$ In this study, we hypothesised that premature infants were more susceptible to disordered lung healing because of reduced neutrophil apoptosis. Grigg et al noted apoptotic neutrophils at single time points in the lungs of eight mechanically ventilated infants. Their results, however, did not correlate with pulmonary outcome. We examined ventilated infants on multiple occasions over the first week of life and showed that the percentage of apoptotic neutrophils in tracheal fluid did not increase above $0.25 \%$ of the total neutrophil count until after the fourth day of life in infants with CLD. Our results suggest that this relative delay may expose vulnerable infants to continued neutrophil mediated lung injury. ${ }^{5}$ Periods of specimen analysis and collection were divided into two periods of the first week of life-that is, days 1-4 and days 5-7-because fewer tracheal fluid specimens were collected after the first 4 days of life in non-CLD preterm and term infants and because analysis of neutrophil counts in the later part of the week would have allowed us to capture the persistent pulmonary neutrophilia characteristic of developing CLD. ${ }^{24}$

IL10 is currently the only known endogenous factor that promotes neutrophil apoptosis. ${ }^{9}$ Exogenous IL10 attenuates pulmonary inflammation in experimental animals by accelerating neutrophil apoptosis and decreasing neutrophil influx. ${ }^{92}$ Various forms of chronic and severe pulmonary inflammatory disorders are associated with low levels of IL10, including cystic fibrosis, asthma, and fatal acute respiratory distress syndromes. ${ }^{26-28}$ IL10 levels are also reduced early in life in infants who develop CLD of prematurity. ${ }^{29}{ }^{30} \mathrm{McColm}$ et $a l^{12}$ studied 11 infants less than 30 weeks gestation, nine of whom developed CLD. Detectable IL10 was found in 57\% of the tracheal fluid specimens regardless of subsequent CLD development. However, only two infants without CLD were studied, and higher gestation infants were not studied by their particular laboratory methods for comparison. We have shown (in a concurrent study) that, although IL10 is predominantly governed by gestation, more mature infants who were not expected to develop CLD also had minimal IL10 in their lung fluid. For example, a 29 week male infant needing only minimal ventilation for apnoea and a 31 week male infant with mild HMD had neither IL10 or apoptotic neutrophils in any of their week 1 tracheal fluid specimens. Nevertheless, higher levels of ILlo produced by more mature infants may have contributed to lung healing by constraining neutrophil activity, as evidenced by increased levels of apoptosis. This study, however, failed to show a positive correlation between IL10 and neutrophil apoptosis.

Other cytokines, however, also significantly influence neutrophil longevity, and it is difficult to determine from our study whether a causal relation exists between endogenous IL10 and neutrophil apoptosis. Proinflammatory cytokines such as IL8 and IL6 retard neutrophil apoptosis and are also increased in the lung fluid of CLD prone infants. ${ }^{17}{ }^{32-34}$ There were also infants in our study who did not develop CLD despite having neither high levels of IL10 nor apoptotic neutrophils. Most of these infants, nevertheless, were more mature, and the pulmonary protective value of IL10 may be only significant in the most at risk-that is, the extremely preterm population.

The peak frequency of neutrophil apoptosis occurs 6-72 hours after pulmonary injury, ${ }^{3}$ and previous observations have shown that neutrophil apoptosis and subsequent phagocytosis by primed macrophages is a rapid process; neither neutrophils nor their cytoplasmic contents are recognisable as soon as 30 minutes after engulfment. ${ }^{6}$ Optimal in vitro identification of neutrophil apoptosis in our study may have been limited by various clinical constraints (such as laboratory availability and tracheal suction timing). Non-CLD specimens in our study were less complete than CLD specimens because most of these infants had been extubated by the end of the week. Therefore, we do not have a complete profile of infants without CLD throughout the first week, and IL10 and neutrophil apoptosis may have been evident in these infants if sampling times had differed. Even so, our findings describe a novel lack of neutrophil apoptosis and IL10 in CLD specimens compared with non-CLD specimens during the first 4 days of life.

We identified neutrophil apoptosis by light microscopy in a similar fashion to Savill et $a l^{6}$ by noting shrinkage of the entire neutrophil and condensation of the nuclear chromatin into a rounded lobe. We also observed some atypical clump-like changes suggested by Ishii $\mathrm{et}_{\mathrm{al}}{ }^{8}$ to be representative of an early part of the apoptotic process.

Exogenous factors such as commonly used medications may modulate the rate of neutrophil apoptosis. Erythromycin $^{35}$ and theophylline ${ }^{36}$ accelerate, whereas glucocorticoids retard, ${ }^{37}$ neutrophil apoptosis. The antiinflammatory properties of glucocorticoids are well known. Despite retarding neutrophil apoptosis, glucocorticoids promote macrophage phagocytosis of apoptotic white cells. ${ }^{38}$ However, none of the infants in our study were treated with glucocorticoids during week 1 , and most of the infants without CLD (term and preterm) did not receive theophylline until immediately before extubation. We therefore cannot conclude that the difference in the frequency of apoptosis between the groups of infants in our study was influenced in any way by theophylline or glucocorticoids. Further study is needed to assess the effect that these medications have on early pulmonary inflammation in susceptible preterm infants.

In conclusion, our results show that neutrophil apoptosis increases with gestational maturity and that infants who subsequently developed CLD had negligible amounts of apoptosis and IL10. However, the interplay of factors controlling pulmonary inflammation is complex and probably not dominated by any one cytokine. IL10 has anti-inflammatory properties that 
may have the potential to mitigate the neutrophil mediated disorganised pulmonary healing and repair characteristic of CLD. Our study supports the observation of Jones et al ${ }^{29}$ showing low IL10 in tracheal fluid in CLD prone infants. As glucocorticoids, with all their numerous side effects, are the only current relatively beneficial treatment for CLD, further investigation into ways of modulating the inflammatory profile of the preterm lung with novel anti-inflammatory treatments during the first week of life may eventually help to prevent and treat CLD.

\section{ACKNOWLEDGEMENTS}

This research study was supported by grants from the Sydney Children's Hospital Foundation and the Royal Hospital for Women Foundation.

\section{Authors' affiliations}

J Oei, K Lui, H Wang, R Henry, School of Women's and Children's Health, University of New South Wales, Sydney, Australia

J Oei, K Lui, Department of Newborn Care, Royal Hospital for Women, Sydney, Australia

H Wang, Paediatric Research Laboratory, Sydney Children's Hospital, Sydney, Australia

\section{REFERENCES}

1 Robertson B. The evolution of neonatal respiratory distress syndrome into chronic lung disease. Eur Respir J 1989;2(suppl 3):33S-37S.

2 Deneke SM, Fanburg BL. Normobaric oxygen toxicity of the lung. $N$ Engl J Med 1980;303:76-86.

3 Cox G, Crossley J, Xing Z. Macrophage engulfment of apoptotic neutrophils contributes to the resolution of acute pulmonary inflammation in vivo. Am J Respir Cell Mol Biol 1995;12:232-23.

4 Ogden B, Murphy S, Saunders G, et al. Neonatal lung neutrophils and elastast/proteinase inhibitor im, balance. Am Rev Respir Dis 1984; 130:917-821

5 Smith JA. Neutrophils, host defense and inflammation: a double-edged sword. J Leukoc Biol 1994;56:672-84.

6 Savill JS, Wyllie AH, Henson JE, et al. Macrophage phagocytosis of aging neutrophils in inflammation. J Clin Invest 1989:83:865-75.

7 Grigg JM, Savill JS, Sarraf C, et al. Neutrophil apoptosis and clearance from neonatal lungs. Lancet 1991;338:720-2.

8 Ishii Y, Hashimoto K, Nomura A, et al. Elimination of neutrophils by apoptosis during resolution of acute pulmonary inflammation in rats. Lung 1998; 176:89-98.

9 Cox G. II-10 enhances resolution of pulmonary inflammation in vivo by promoting apoptosis of neutrophils. Am J Physiol 1996;271:L566-71.

10 Shennan AT, Dunn MS, Ohlssono A, et al. Abnormal pulmonary outcomes in premature infants. Prediction from oxygen requirement in the neonatal period. Pediatrics 1988;82:527-32.

11 Henderson-Smart DJ, Davis PG. Prophylactic methylxanthine for extubation in preterm infants. Cochrane Database Syst Rev 2000;(2):CD000139

12 McColm JR, Stenson BJ, Biermasz N, et al. Measurement of interleukin 10 in bronchoalveolar lavage from preterm ventilated infants. Arch Dis Child Fetal Neonatal Ed 2000;82:F156-9.

13 Saraste A. Morphologic criteria and detection of apoptosis. Herz 1999;24:189-95.

14 NHLBI Workshop Summary. Assessment of lung function and dysfunction in studies of infants and children. Am Rev Respir Dis 1993;148:1105-8.

15 Wang P, Wu P, Anthes JC, et al. Interleukin-10 inhibits interleukin-8 production in human neutrophils. Blood 1994;83:2678-83.
16 Cassatella MA, Meda L, Bonora M. IL-10 inhibits the release of proinflammatory cytokines from human polymorphonuclear leukocytes. Evidence for an autocrine role of tumor necrosis factor and IL-1 $\beta$ in mediating the production of IL-8 triggered by lipopolysaccharide. J Exp Med 1993;178:2207-11.

17 Kunkel SL, Standiford T, Kasahara K, et al. Interlukin-8: the major neutrophil chemotactic factor in the lung. Exp Lung Res 1991;17:17-23.

18 Dunican AL, Leuenroth SJ, Grutkoski P, et al. TNFalpha-induced suppression of PMN apoptosis is mediated through interleukin-8 production. Shock 2000;14:288-9

19 Oei J, Lui K, Wang H, et al. Early anti- and pro-inflammatory balance in premature newborn lungs during mechanical ventilation: a pilot study [abstract]. J Paediatr Child Health 2000;36:A4.

20 Whyte MKB, Meagher LC, MacDermot J, et al. Impairment of function in aging neutrophils is associated with apoptosis. J Immuno 1993; 150:5124-34.

21 Dransfield IS, Stocks C, Haslett C. Regulation of cell adhesion molecule expression and function associated with neutrophil apoptosis. Blood 1995;85:3264-73.

22 Stern M, Savill J, Haslett C. Human monocyte-derived macrophage phagocytosis of senescent eosinophils undergoing apoptosis: mediation by $\alpha v \beta 3 / C D 36 /$ thrombospondin recognition mechanism and lack of phlogistic response. Am J Pathol 1996;149:911-21.

23 Hussain N, Wu F, Zhu L, et al. Neutrophil apoptosis during the development and resolution of oleic acid-induced acute lung injury in the rat. Am J Respir Cell Mol Biol 1998; 19:867-74.

24 Arnon S, Grigg J, Silverman M. Pulmonary inflammatory cells in ventilated preterm infants: effect of surfactant treatment. Arch Dis Child 1993;69:44-8.

25 Chmiel JF, Konstan MW, Knesebeck JE, et al. IL-10 attenuates excessive inflammation in chronic pseudomonas infection in mice. Am J Respir Crit Care Med 1999;160:2040-7

26 Bonfield TL, Panuska JR, Konstan P, et al. Inflammatory cytokines in cystic fibrosis lungs. Am J Respir Crit Care Med 1995;13:257-61.

27 Borish L, Aarons A, Rumbyrt $\mathrm{P}$, et al. Interleukin-10 regulation in normal subjects and patients with asthma. J Allergy Clin Immunol 1996;97:1288-96.

28 Donnelly SC, Streiter RM, Reid RT, et al. The association between mortality rates and decreased concentrations of interleukin- 10 and interleukin-1 receptor antagonist in the lung fluids of patients with respiratory distress syndrome. Ann Intern Med 1996;125:191-6.

29 Jones CA, Cayabyab RG, Kwong KYC, et al. Undetectable interleukin (IL)-10 and persistent IL-8 expression in hyaline membrane disease: a possible developmental basis for the predisposition to chronic lung inflammation in preterm newborns. Pediatr Res 1996:39:966-75

30 Jonsson B, Li YH, Noack G, et al. Downregulatory cytokines in tracheobronchial aspirate fluid from infants with chronic lung disease of prematurity. Acta Paediatr 2000;89:1375-80.

31 Groneck P, Gotze-Speer, Opperman M, et al. Association of pulmonary inflammation and increased microvascular permeability during the development of bronchopulmonary dysplasia: a sequential analysis of inflammatory mediators in respiratory fluids of high-risk preterm neonates. Pediatrics 1994:93;712-18.

32 Jonsson B, Tullus K, Brauner A, et al. Early increase of TNFa and IL-6 in tracheobronchial aspirate fluid indicator of subsequent chronic lung disease in preterm infants. Arch Dis Child Fetal Neonatal Ed 1997;77:F198-201.

33 Biffl WL, Moore EE, Moore FA, et al. Interleukin-6 delays neutrophil apoptosis. Arch Surg 1996;131:24-9.

34 Munshi UK, Niu JO, Siddiq MM, et al. Elevation of interleukin-8 and interleukin- 6 precedes the influx of neutrophils in tracheal aspirates from preterm infants who develop bronchopulmonary dysplasia. Pediatr Pulmonol 1997;24:331-6.

35 Aoshiba K, Nagai A, Konno K. Erythromycin shortens neutrophil surviva by accelerating apoptosis. Antimicrob Agents Chemother by accelerating a

36 Yasui K, Hu B, Nakazawa T, et al. Theophylline accelerates human granulocyte apoptosis not via phosphodiesterase inhibition. J Clin Invest 1997;100:1677-84.

37 Cox G. Glucocorticoid treatment inhibits apoptosis in human neutrophils. J Immunol 1995;154:4719-25

38 Liu Y, Cousin JM, Hughes J, et al. Glucocorticoids promote nonphlogistic phagocytosis of apoptotic leukocytes. J Immunol 1999:162;3639-46. 